\title{
Global and Local Oriented Gabor Texture Histogram for Person Re-identification
}

\author{
Elango Poongothai ${ }^{1}$ \\ https://orcid.org/0000-0002-9616-7045
}

\section{Andavar Suruliandi ${ }^{2}$}

${ }^{1}$ Manonmaniam Sundaranar University, Department of Computer Science and Engineering, Tirunelveli, Tamilnadu, India; ${ }^{2}$ Manonmaniam Sundaranar University, Department of Computer Science and Engineering, Tirunelveli, Tamilnadu, India.

Received: 2018.01.02; Accepted: 2019.07.08.

* Correspondence: poongothai.rp@gmail.com; Tel.: +91-9677254144

\section{HIGHLIGHTS}

- Novel feature extraction method for person re-identification.

- Texture and shape based feature extraction.

- Global and local feature extraction.

- Performance analysis for different person re-identification issues.

\begin{abstract}
Automated person re-identification is a key process in global distributed camera systems. This paper proposes a new feature, the Global and Local-Oriented Gabor Texture Histogram (GLOGTH), for person re-identification. GLOGTH is a combination of the local texture and global structure information of a given input image. This feature aims at representing the human appearance traits with low-dimensional feature extraction. The proposed feature extracts the texture information of input images based on the orientation of the weighted gradient from the global representation. In GLOGTH, the principal orientation is determined by the gradient of the pixels. Based on the principal orientation, the Gabor feature is extracted and imbues GLOGTH with the strong ability to express edge information, apart from making it robust to lighting variances. The experimental results acquired from the databases demonstrate that the proposed GLOGTH framework is capable of achieving notable improvements, in many cases reaching higher classification accuracy than traditional frameworks.
\end{abstract}


Keywords: Person Re-identification, Texture Feature, Global feature, Local feature

\section{INTRODUCTION}

Person re-identification is recognizing and matching a person from different locations, at different times, in multiple cameras using the soft biometrics. It has become an active research topic in the area of computer vision applications. The most frequently used appearance feature descriptors on feature analysis for person identification include the texture, colour and geometrical shape of a person's body. The well performed texture closely matches the target with accurate information. Of these, SIFT [1], Histograms of Oriented Gradients (HOG) [2,3] Gabor [4], and LBP [5-9], are the most well known, and have emerged as widely applied features for tracking a person and for retrieval. These descriptors work on the principle of taking raw information from the magnitude and orientations of pixel. The existing research analysis [18-21] reveals that most researchers preferred the Gabor and HOG as simple yet powerful features, extensively used in diverse fields for texture representation. So, by combing Gabor and Histogram Oriented Gradients (HOG), new feature GLOGTH is proposed for person reidentification. The performance of this feature is reviewed in terms of issues such as illumination, occlusion and pose variation. In order to demonstrate the effectiveness of the proposed GLOGTH method, comparative experiments were performed with the results of other state-of-the-art features. It proved that the proposed feature performs well with high accuracy in terms of target matching.

\section{Related work}

The Gabor and LBP [1-5] are the most commonly used texture features for image analysis and retrieval. Jung et al [9] proposed a method aimed at representing human facial traits with low-dimensional feature extraction using an orthogonal linear discriminant analysis (OLDA). Likewise for global and local representation, Feng et al [10] contributed a structural difference histogram representation by fusing the global structure using the segmented structure pattern (SSP), the local texture using the refined LBP (RLBP), and the neighbourdifference pattern (NDP) for texture classification. Particularly, the segmented structure containing the global contour information of an image texture is first constructed to compute its SSP histogram feature. Simultaneously, the RLBP is defined to represent the local texture information. Symmetry and uniformity structural regularization constraints were proposed by Ren et al [11] to optimize the LBP structure. These two constraints are created by predefined local binary pattern structures, following human prior knowledge in designing the structure of the LBP. 
An efficient, novel method proposed by Zeng et al [12] for person re-identification is based on the Mahalanobis distance feature that exhibits extraordinary discriminative power. To enhance the overall performance, the lightweight PCA is cascaded with the Keep It Simple and Straightforward (KISS) learning metric. Yoga Meena [13] et al proposed a classification algorithm to classify objects with the presence of shadows and occlusion present in the video. The Gabor-based Gaussian mixture model is used to eliminate shadows and subtract the background in the images concerned Chai et al [14] introduced a novel Gabor ordinal measure (GOM) for facial feature extraction by integrating Gabor features with ordinal measures. Ortiz et al [15] proposed a novel normalization scheme to minimize hallucinatory effects. It is built, based on the results of a detailed analysis of gradient distribution by adding an extra bit with a special value to increase the distinctiveness of HOG. To this end, Kawai et al [16] employed a spatio-temporal histogram of oriented gradients based on gradient-based motion, gait and shape features that concatenate motion changes with shape features. Corvee et al [17] proposed a dominant color descriptors based appearance model with Haar-like features.

According to the literature surveyed, appearance-based methods are more appropriate for person re-identification because of the short time involved in the entire process. So far, no research has been carried out to evaluate the performance of global and local description as a single feature under real-time challenges in person identification. The remaining part of the paper deals with the different sections and their contents. Section 2 reports on the methodology with the feature extraction process of the GLOGTH descriptor. Section 3 deals with the experimental results and discussions. Section 4 concludes the proposed work.

\section{Motivation of the proposed work}

Among all the local feature models, texture descriptors plays an important role in recognizing person because of its shape-free feature representation. Though variations in pose and illumination directly affect texture and shape features, the availability and potential of the texture features are excessive in the person's images. From the literature reviewed, it is noted that texture as a stand-alone feature does not work well for person re-identification, and must be combined with colour or shape feature for improved performance. Following an in-depth analysis, researchers have since considered texture and shape features for target matching, given their unique characteristics in terms of performance in certain conditions. As these texture and shape features are computed on the intensity image, they play a critical role in establishing correspondence when the colour information gets degraded under drastic illumination changes and/or change of camera settings. Motivated by this fact, a new feature GLOGTH is proposed 
here with shape and texture combination to detect persons, while satisfying the criteria of distinctiveness and robustness.

\section{METHODOLOGY}

\section{Outline of the work}

The entire processing of person re-identification is illustrated in Fig.1.

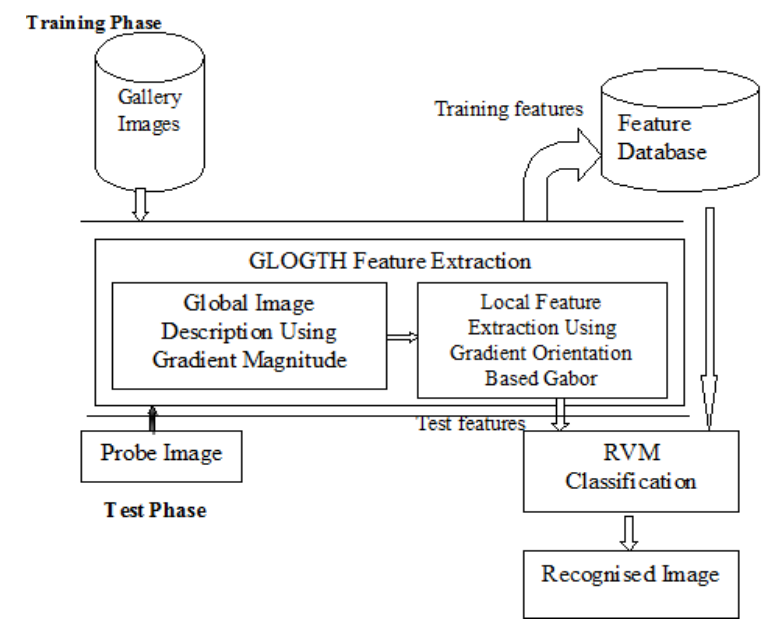

\section{Fig. 1. Process of Proposed GLOGTH Feature Extraction}

\section{GLOGTH Feature Extraction}

\section{Global representation}

The goal of texture analysis is to extract local region information from the image. For global feature extraction, information is extracted from the whole region of the image. In order to build rotation-invariant features possessing distinctiveness, a feature is proposed by applying the concept of calculating the gradient-based structure to describe the distribution of the holistic orientations and to compensate for the global-level rotation. The Gabor-based texture describes the distribution of the local texture for each orientation.

\section{Step 1}

The person image is given as input I. The image gradient is computed from the input image, based on the first-order partial derivative,

$$
\mathrm{I}_{\mathrm{Grad}}(\mathrm{x}, \mathrm{y})=\left(\frac{\partial \mathrm{I}}{\partial \mathrm{x}}, \frac{\partial \mathrm{I}}{\partial \mathrm{y}}\right)
$$


where $\mathrm{x}$ is a pixel value in a horizontal direction; and $\frac{\partial \mathrm{I}}{\partial \mathrm{x}}=2 \mathrm{xy} ; \mathrm{y}$ is a pixel value in a vertical direction, and $\frac{\partial \mathrm{I}}{\partial y}=\mathrm{x}^{2}$. From the gradient values, the gradient magnitude $\mathrm{m}(\mathrm{x}, \mathrm{y})$ of each pixel $\mathrm{p}(\mathrm{x}, \mathrm{y})$ is calculated using Equation (2).

$$
\mathrm{m}(\mathrm{x}, \mathrm{y})=\sqrt{\left(\frac{\partial \mathrm{I}}{\partial \mathrm{x}}\right)^{2}+\left(\frac{\partial \mathrm{I}}{\partial \mathrm{y}}\right)^{2}}
$$

The gradient orientation of each pixel is calculated using Equation (3), and evenly discretized across $0^{\circ}-360^{\circ}$.

$$
\text { Orientation } \theta=\tan ^{-1}\left(\mathrm{I}_{\mathrm{Grad}}(\mathrm{x}, \mathrm{y})\right)
$$

The image is represented with gradient orientations of the image, with $\mathrm{k}$ bins covering the $360^{\circ}$ range of orientations. Suppose the texture image is $\mathrm{M} \times \mathrm{N}$, after computing the image gradient and discretizing the orientation for each pixel, the entire image is represented by building a histogram, as follows:

$$
\mathrm{H}(\theta)=\sum_{\mathrm{x}=1}^{\mathrm{M}} \sum_{\mathrm{y}=1}^{\mathrm{N}} \mathrm{m}(\mathrm{x}, \mathrm{y}) \mathrm{f}\left(\theta_{\mathrm{k}} \theta_{\mathrm{p}}\right), \quad \mathrm{k} \in[1, \mathrm{k}]
$$

where $\theta_{p}$ is the quantified orientation of each pixel $p(x, y) ; k$ is the number of bins of the histogram, and $m(x, y)$ is the gradient magnitude of the pixel $p(x, y)$. Each sample added to the histogram is weighed, based on its gradient magnitude.

\section{Step 2}

Once the global representation has been completed by representing the gradient orientation and magnitude of each pixel, the image is divided into four equal divisions of equal size; upper right, upper left, lower right and lower left. The image is divided into 4 blocks, each of which is further divided into 32 cells sized $8 * 4$. After dividing the image into cells, the gradient orientation from each pixel is extracted from the image, and the orientation will be calculated for the pixels from every block. For each block, 32 orientations are calculated and ordered in the histogram. The orientations ordered to the histogram are weighed, based on their gradient magnitude. From every cell the principle orientation is calculated based on the highest weight of the histogram. Other orientations are ordered based on the principle orientations. All the orientation bins of the histogram are shifted until the principal orientation places its first position.

Thereafter, the Gabor filter is applied using the 32 gradient orientations of the histogram. The Gabor feature is calculated for 4 scales and 32 orientations, using the equations shown below.

For complex values 


$$
\mathrm{g}(\mathrm{x}, \mathrm{y} ; \lambda, \theta, \varphi, \sigma, \gamma)=\exp \left(-\frac{\mathrm{x}^{\prime 2}+\gamma^{2} \mathrm{y}^{\prime 2}}{2 \sigma^{2}}\right) \exp \left(\mathrm{i}\left(2 \pi \frac{\mathrm{x}^{\prime}}{\lambda}+\varphi\right)\right)
$$

For real values

$$
\mathrm{g}(\mathrm{x}, \mathrm{y} ; \lambda, \theta, \varphi, \gamma, \sigma)=\exp \left(-\frac{\mathrm{x}^{\prime 2}+\gamma^{2} \mathrm{y}^{\prime 2}}{2 \sigma^{2}}\right) \cos \left(2 \pi \frac{\mathrm{x}^{\prime}}{\lambda}+\varphi\right)
$$

For imaginary values

$$
\mathrm{g}(\mathrm{x}, \mathrm{y} ; \lambda, \theta, \varphi, \gamma, \sigma)=\exp \left(-\frac{\mathrm{x}^{\prime 2}+\gamma^{2} \mathrm{y}^{\prime 2}}{2 \sigma^{2}}\right) \sin \left(2 \pi \frac{\mathrm{x}^{\prime}}{\lambda}+\varphi\right)
$$

Here,

$$
\begin{aligned}
& x^{\prime}=x \cos \theta+y \sin \theta \\
& y^{\prime}=-x \sin \theta+y \cos \theta
\end{aligned}
$$

where $\lambda$ represents the wavelength of the sinusoidal factor, and $\theta$ represents the orientation bin of the normal to the parallel stripes. Its value is determined in degrees between 0 and $360, \varphi$ is the phase offset, $\sigma$ is the standard deviation of the Gaussian envelope and $\gamma$ is the spatial aspect ratio of the Gabor function. Finally, the Gabor feature is built for each bin k. The final feature is determined as follows:

$$
\text { GLOGTH }=\left\{\operatorname{GLOGT}^{\theta_{1}}, \operatorname{GLOGT}^{\theta_{2}}, \ldots \ldots \text { GLOGT }^{\theta_{k}}\right\}
$$

\section{RVM (Ranking Vector Machine) classification}

RVM is a ranking based approach widely used for image retrieval applications, particularly in computer vision, where it has been used for an array of applications such as face recognition, personal identification, background subtraction, and hand gesture detection. This is a regression-based machine learning method that provides the probabilistic classification of the given input vectors. It classifies the input vector based on the ranking vector.

$$
\mathrm{k}\left(\mathrm{x}, \mathrm{x}^{\prime}\right)=\sum_{\mathrm{j}=1}^{\mathrm{N}} \frac{1}{\sigma_{\mathrm{j}}} \varphi\left(\mathrm{x}, \mathrm{x}_{\mathrm{j}}\right) \varphi\left(\mathrm{x}^{\prime}, \mathrm{x}_{\mathrm{j}}\right)
$$

Where $\varphi$ is the kernel function used to map the feature between two images, $\sigma_{j}$ the variance of the weighting vector $(0$ and 1$)$ and $x_{1}, x_{2}, \ldots \ldots . . . x_{N}$, the input feature vectors of the training set. The RVM avoids the set of free parameters that usually require for cross-validationbased post-optimizations. However RVMs use an expectation maximization like learning method and classifies images based on the local minima, which are quarantined to find the 
global minima. Based on the global minima, it ranks the image so it might be matched. The topranked images of the given target are considered closed matches.

\section{Algorithm for Feature Extraction for the Re-identification Process}

a. Read the input image.

b. Calculate the gradient value of each pixel using Equation 1.

c. Calculate the gradient magnitude using Equation 2.

d. Calculate the gradient orientation of each pixel using Equation 3.

e. Represent the image in the form of gradient magnitude and orientation

f. Divide the image into four blocks

g. Divide each block into 32 cells.

h. Weigh the orientation of each pixel by its magnitude.

i. Calculate the principle orientation from the sample orientations based on highest weight.

j. Order the holistic orientation, based on the principle orientation.

k. $\quad$ Compute the Gabor features for 32 orientations using Equations 4.5 to 4.9 .

1. Repeat steps 6 to 12 for all blocks.

m. Compute the HSV values for the input image.

n. Store the GLOGT and HSV as a feature vector in the training database.

Test Phase

1. Extract features for the given test image using the feature extraction procedure from steps 1 to 13 , since the training phase is in operation.

2. Classify the image using the k-NN classification

\section{EXPERIMENTAL RESULTS AND ANALYSIS}

We extensively performed the series of experiments to evaluate the effectiveness of the proposed GLOGTH feature. We carried out the evaluation on four benchmark datasets [22]. The performance of proposed feature is evaluated in the dataset namely Viper, CAVIAR, PRID and $\mathrm{ETHZ}[9]$ by using the metrics called identity fragmentation $\left(F_{\mathrm{ID}}\right)$, classifier coverage $(\mathrm{C})$, unknown rate (UR) which are introduced by Kenk et al [14]. 


\section{Identification accuracy of GLOGTH for different window Size}

It is important to evaluate the significance of the feature description of an image by varying the window size. This is because the size of the window greatly impacts the accuracy of any feature description. The HOG extracts the full structure of the image, It does not depend on the window size. From HOG we observe that the full HOG detection does not support the highest match and, further, also increases the feature dimension. For our proposed GLOGTH feature, however, we consider local texture description as well. It is, therefore, most important to evaluate the local texture information with different window sizes.

Normally, given that human images are bigger in terms of size rather than width, the images from the dataset are partitioned into grids of rectangular spatial cells. The four classes of blocks are of sizes $64^{*} 32,32^{*} 16,16^{\star} 8$, and $8^{*} 4$. The accuracy for every dataset is shown in Table 1. Here, the row represents datasets and the column represents accuracy for various window sizes.

Table 1. Identification Accuracy of Proposed Feature for different Window Size

\begin{tabular}{|c|l|l|l|l|}
\hline $\begin{array}{c}\text { Window } \\
\text { Size } \\
\text { Vs }\end{array}$ & \multicolumn{4}{|c|}{ Accuracy in \% } \\
\cline { 2 - 5 } Dataset & $\begin{array}{l}\text { 64X32 } \\
\text { (4 cell) }\end{array}$ & $\begin{array}{l}\text { 32X16 } \\
\text { (8 cell) }\end{array}$ & $\begin{array}{l}\text { 16X8 } \\
(16 \text { cell) })\end{array}$ & $\begin{array}{l}\mathbf{8 X 4} \\
(32 \text { cell })\end{array}$ \\
\hline Viper & 87.2 & 92.6 & 96.8 & 99.4 \\
\hline ETHZ & 92.1 & 95.7 & 98.6 & 99.7 \\
\hline PRid & 82.1 & 86.7 & 91.5 & 96.8 \\
\hline Caviar & 76.4 & 83.4 & 89.6 & 94.5 \\
\hline
\end{tabular}

In Table 1, a slight improvement is observed when considering the maximum number of cells needed to calculate the gradient orientation. Accuracy increases as more cells are considered in a window. Table 1 shows that the best performance is always obtained with a window size of $8 \times 4$.

\section{Performance of GLOGTH for different orientation bins for different datasets}

The Viper, Ethz, Caviar and PRid datasets are used in the experiments to evaluate the performance of the proposed feature by varying the orientation bins, and the results are shown 
in Fig. 2. The number of bins $\mathrm{K}=10,20,30$, and 32 were set from the global structure histogram. Recognition accuracies versus different orientation bins on different datasets are illustrated in Fig. 2 (a-d), and the best recognition accuracy may be obtained when $\mathrm{K}$ is equal to 32 on the Viper and Caviar databases. Totally $4{ }^{\star} 32=128$ bins used for GLOGTH 8, 4 window. Fig. 2 summarizes the upshot of the proposed feature on the overall detection performance for different bins.

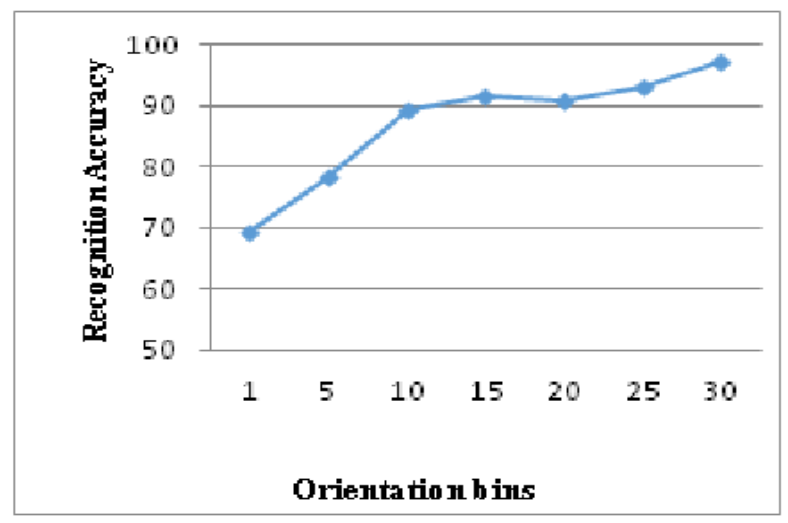

a) VIPER

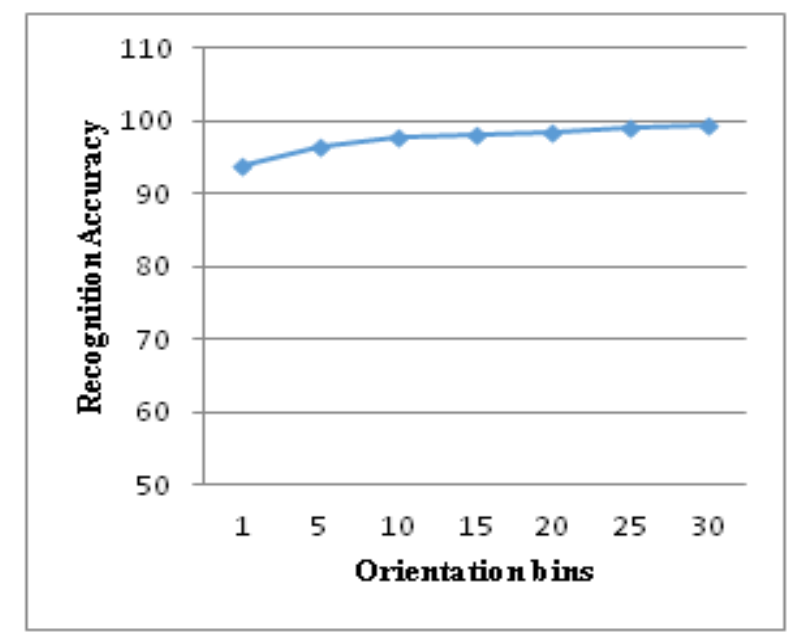

c) CAVIAR

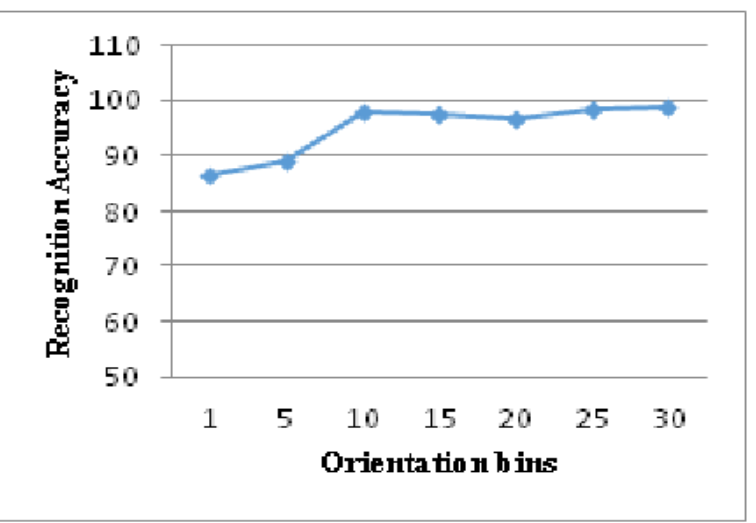

b) ETHZ

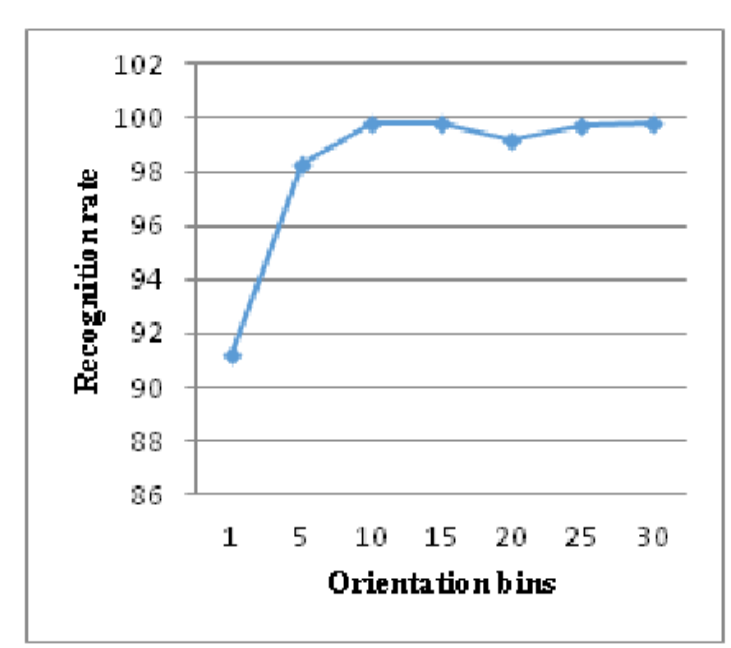

d) PRID

Fig. 2(a-d) Recognition Accuracy of proposed feature for different orientation bins for different datasets

Fig. 2(d) shows that the performance can be improved significantly by increasing the number of orientation bins up to about 25 , though there is little variation beyond the by noise being incorporated into the image, as a result of segmentation errors and strong lighting variations. Fig. 2(b) shows, in most cases, that GLOGTH achieves much better result when the number of orientation bins increases. In line with our analysis in Section 2.2, GLOGTH uses 
gradient magnitude as the weight of the histogram, making it robust to rotation invariants. In addition, GLOGTH has the capacity to express edge information strongly.

\section{Identification accuracy of GLOTH for different angle orientations}

Fine orientation is essential for any feature extraction to perform well, and we undertake to assess the proposed feature for 8 different orientation angles: 30, 90, 135, 180, 225, 270, 315 , and 360 . The angle of the image is calculated, based on the method in [23]. Subsequently, we match the target with various angle orientations using the proposed feature. The identification rate for all the angle variations is shown in Table 2.

As illustrated in Table.2, identification accuracy reaches the highest for all the angle variations. From these results, we note that GLOGTH is robust to rotation invariants. Since it supports the property of rotation invariance, it detects edge information as well. We see this in Table 2, where the accuracy is high for all the datasets with rotation variations. It works well at handling inter-person similarities as well as intra-person variations for face images

Table 2. Identification Accuracy of Proposed Feature for Angle Orientation

\begin{tabular}{|c|c|c|c|c|}
\hline \multirow{3}{*}{ Angle Orientation } & \multicolumn{4}{|c|}{ Accuracy in \% } \\
\hline & \multicolumn{4}{|c|}{ Datasets } \\
\hline & Viper & ETHZ & PRid & Caviar \\
\hline $0-30$ & 83.5 & 92.7 & 96.5 & 88 \\
\hline $45-90$ & 93.1 & 93.3 & 98.6 & 79 \\
\hline $90-135$ & 92.6 & 94.8 & 99.7 & 98.4 \\
\hline $135-180$ & 88.4 & 87.4 & 93.4 & 91.2 \\
\hline $180-225$ & 91.4 & 89.5 & 97.4 & 98.2 \\
\hline $225-270$ & 92.4 & 90.6 & 97.7 & 98.4 \\
\hline $270-315$ & 86.5 & 79.3 & 83.0 & 89.1 \\
\hline $315-360$ & 85.5 & 84.3 & 78.5 & 68.3 \\
\hline
\end{tabular}

\section{Performance of the proposed GLOTH for different issues}

To investigate the robustness of the proposed GLOGTH, an evaluation is carried out using the performance metrics Fid, UR, and C. Recognition of person images under different lighting conditions is a challenge in computer vision, with changes in illumination greatly 
affecting classification. For humans, the wide range of clothing and background colours presumably makes the signs of contrasts uninformative.

To analyze the effectiveness of the proposed method, the sample results for pose variation and occlusion changes are shown in it is evident that the proposed GLOGTH performs well in terms of the issues cited above. The result analysis is shown in Tables 3,4 and 5. Here, except for $F_{i d}$, all the metrics represent higher accuracies. The lowest $F_{\text {id }}$ enhances the system's performance until it registers a high.

Table.3 Performance of Proposed Method for Illumination

\begin{tabular}{|l|l|l|l|l|}
\hline \multirow{2}{*}{\begin{tabular}{l} 
Merformance \\
\cline { 2 - 5 }
\end{tabular}} & \multicolumn{4}{|c|}{ Top Rank Matching } \\
\cline { 2 - 5 } & Rank 1 & Rank 5 & Rank10 & Rank 20 \\
C & 0.18 & 0.12 & 0.00 & 0.00 \\
Fld $_{\text {Precision }}$ & 0.93 & 0.98 & 0.97 & 0.99 \\
Recall & 0.91 & 0.93 & 0.98 & 0.99 \\
Identification & 0.74 & 0.86 & 0.88 & 0.87 \\
Rate & 0.89 & 0.91 & 0.96 & 0.98 \\
\hline
\end{tabular}

Table.4 Performance of Proposed Method for Occlusion Changes

\begin{tabular}{|l|l|l|l|l|}
\hline \multirow{2}{*}{ Merformance } & \multicolumn{4}{|c|}{ Top Rank Matching } \\
\cline { 2 - 5 } & Rank 1 & Rank 5 & Rank10 & Rank 20 \\
\hline UN & 0.22 & 0.19 & 0.16 & 0.06 \\
C & 0.94 & 0.96 & 0.96 & 0.98 \\
F Id $_{\text {Precision }}$ & 0.87 & 0.89 & 0.96 & 0.98 \\
Recall & 0.82 & 0.86 & 0.88 & 0.98 \\
Identification & 0.87 & 0.91 & 0.97 & 0.99 \\
Rate & 0.94 & 0.97 & 0.99 & 0.99 \\
\hline
\end{tabular}

Table.5 Performance of Proposed Method for Pose Variation

\begin{tabular}{|l|l|l|l|l|}
\hline Performance & \multicolumn{4}{|c|}{ Top Rank Matching } \\
\cline { 2 - 5 } Metrics & Rank 1 & Rank 5 & Rank10 & Rank 20 \\
\hline
\end{tabular}




\begin{tabular}{|l|l|l|l|l|}
\hline UN & 0.32 & 0.26 & 0.18 & 0.12 \\
CC & 0.93 & 0.93 & 0.97 & 0.96 \\
Fld $_{\text {Precision }}$ & 0.88 & 0.91 & 0.96 & 0.99 \\
Recall & 0.92 & 0.96 & 0.98 & 0.97 \\
Identification & 0.77 & 0.81 & 0.87 & 0.96 \\
$\quad$ Rate & 0.88 & 0.93 & 0.96 & 0.99 \\
\hline
\end{tabular}

Also, as shown in Tables 3-5, our approach outperformed other approaches at a rank-1 matching rate on the Viper dataset. As depicted in Tables 3,4 and 5, Fid decreases and the other values increase, revealing that the proposed feature performs well by overcoming the challenges caused because of the issues cited above. The proposed approach demonstrated a much better performance because we adopted the local feature and global structure here.

\section{Performance comparison of the proposed GLOGTH feature with the existing features:}

To prove the effectiveness of the proposed method, we compared our proposed GLOGTH feature with the existing features using different re-identification methods. For the purpose of analysis here, we perform our experiment on the Viper dataset and shown in Fig.3.

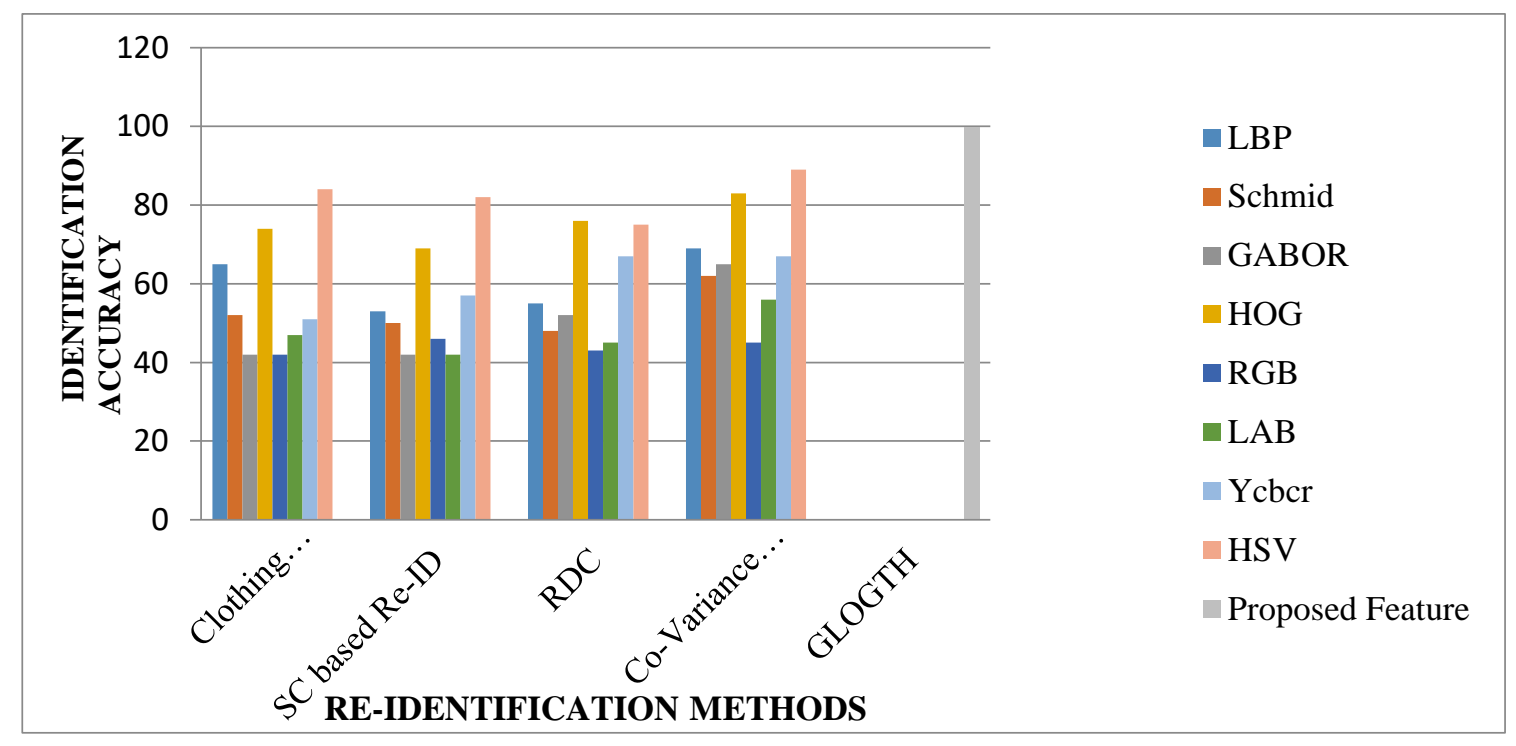

Fig.3 Identification accuracy of proposed feature with Existing features

From the Fig. 3 it is noticed that GLOGTH achieves better and more robust results than state-ofthe-art features [24] such as the LBP (Local Binary pattern), Gabor, Schmid, HOG(Histogram 
Oriented Gradient), Lab, YCbCr and HSV. Given that GLOGTH combines both global structure information and local texture features, and characterizes image information across different directions, it contains abundant information, and so the GLOGTH feature is much more distinctive than others. We conclude that one should use fine derivatives, many orientation bins, and moderately-sized, overlapping descriptor blocks for a fine performance.

\section{CONCLUSION}

In order to represent local texture and global structure information in texture images, a new texture descriptor descriptor, GLOGTH. is proposed in this paper for person reidentification. The HOG feature describes the global structure, and the Gabor feature detects the distribution of local texture patterns using each orientation. First, the principal orientations of the texture image were estimated based on HOG, and according to the principal orientation, the bins were shifted to the initial position to compensate for the rotations of texture images. The proposed GLOGTH efficiently joined the local gradient orientations to the Gabor. The experimental results on four large databases demonstrates that the proposed GLOGTH feature offers a higher degree of identification accuracy than traditional state-of-the-art methods.

\section{References}

1. Zhou, Deyun, Lina Zeng, Junli Liang, and Kun Zhang. "Improved method for SAR image registration based on scale invariant feature transform." IET Radar, Sonar \& Navigation 2016.

2. Hsiao, Shen-Fu, Jun-Mao Chan, and Ching-Hui Wang. "Hardware design of histograms of oriented gradients based on local binary pattern and binarization." In Circuits and Systems (APCCAS), 2016 IEEE Asia Pacific Conference on, pp. 433-435. IEEE, 2016.

3. Yang, H., Wang, X., Zhu, J., Ma, W. and Su, H.,. Resolution adaptive feature extracting and fusing framework for person re-identification. Neurocomputing, 212, pp.65-74, 2016.

4. Wu, S., Chen, Y.C., Li, X., Wu, A.C., You, J.J. and Zheng, W.S., 2016, March. An enhanced deep feature representation for person re-identification. In Applications of Computer Vision (WACV), IEEE Winter Conference on (pp. 1-8). IEEE. 2016

5. Z. Guo, L. Zhang and D. Zhang, Rotation invariant texture classification using LBP variance with global matching, Pattern Recogn. 706-719.

6. 43(3) 2010 Zhang, B., Gao, Y., Zhao, S. and Liu, J., 2010. Local derivative pattern versus local binary pattern: face recognition with high-order local pattern descriptor. IEEE transactions on image processing, 19(2), pp.533-544. 
7. H. Lategahn, S. Gross, T. Stehle and T. Aach, Texture classification by modeling joint distributions of local patterns with Gaussian mixtures, IEEE Trans. Image Process. 19(6), $\quad$ 1548-1557 11, 2010.

8. S. Liao, M. W. K. Law and A. C. S. Chung, Dominant local binary patterns for texture classi' cation, IEEE Trans. Image Process. 18(5) 2009 1107-1118.

9. T. Ojala, M. Pietikainen and T. Maenpaa, Multiresolution gray-scale and rotation invariant texture classi ${ }^{-}$cation with local binary patterns, IEEE Trans. Pattern Anal. Mach. Intel. 24(7) 971-987, 2002.

10. Jung, J. Y., Kim, S. W., Yoo, C. H., Park, W. J., \& Ko, S. J. LBP-ferns-based feature extraction for robust facial recognition. IEEE Transactions on Consumer Electroni0cs, 62(4), 446-453, 2016.

11. Feng, Jinwang, Xinliang Liu, Yongsheng Dong, Lingfei Liang, and Jiexin Pu. "Structural difference histogram representation for texture image classification." IET Image Processing 11, no. 2: 118-125, 2016.

12. Ren, Jianfeng, Xudong Jiang, and Junsong Yuan. "LBP-Structure Optimization With Symmetry and Uniformity Regularizations for Scene Classification." IEEE Signal Processing Letters 24, no. 1: 37-41, 2017.

13. Zeng, Mingyong, Zemin Wu, Chang Tian, Lei Zhang, and Xiaolong Zhao. "Person reidentification based on a novel mahalanobis distance feature dominated KISS metric learning." Electronics Letters 52, no. 14: 1223-1225, 2016.

14. Yogameena, B., S. Md Mansoor Roomi, R. Jyothi Priya, S. Raju, and V. Abhaikumar. "People/vehicle classification by recurrent motion of skeleton features." IET computer vision 6, no. 5: 442-450, 2012.

15. Chai, Zhenhua, Zhenan Sun, Heydi Mendez-Vazquez, Ran He, and Tieniu Tan. "Gabor ordinal measures for face recognition." IEEE transactions on information forensics and security 9, no. 1: 14-26, 2014.

16. Ortiz, Javier, Sławomir Bąk, Michał Koperski, and François Brémond. "Minimizing hallucination in histogram of Oriented Gradients." In Advanced Video and Signal Based Surveillance (AVSS), 2015 12th IEEE International Conference on, pp. 1-6. IEEE, 2015.

17. Kawai, Ryo, Yasushi Makihara, Chunsheng Hua, Haruyuki Iwama, and Yasushi Yagi. "Person re-identification using view-dependent score-level fusion of gait and color features." In Pattern Recognition (ICPR), 2012 21st International Conference on, pp. 2694-2697. IEEE, 2012.

18. Corvee, Etienne, Francois Bremond, and Monique Thonnat. "Person re-identification using haar-based and dcd-based signature." In Advanced Video and Signal Based 
Surveillance (AVSS), 2010 Seventh IEEE International Conference on, pp. 1-8. IEEE, 2010.

19. Tao, Dapeng, et al. "Person re-identification by regularized smoothing kiss metric learning." Circuits and Systems for Video Technology, IEEE Transactions on 23.10 : 1675-1685, 2013.

20. Zheng, Wei-Shi, Shaogang Gong, and Tao Xiang. "Reidentification by relative distance comparison." Pattern Analysis and Machine Intelligence, IEEE Transactions on 35.3: 653-668, 2013.

21. Kviatkovsky, Igor, Amit Adam, and Ehud Rivlin. "Colour invariants for person reidentification." Pattern Analysis and Machine Intelligence, IEEE Transactions on 35.7: 1622-1634, 2013.

22. Ma, Bingpeng, Yu Su, and Frédéric Jurie. "Discriminative Image Descriptors for Person Re-identification." Person Re-Identification. Springer London, 23-42, 2014.

23. Vezzani, Roberto, and Rita Cucchiara. "Benchmarking for Person Reidentification." Person Re-Identification. Springer London, 333-349, 2014.

24. Wu, Z., Li, Y. and Radke, R.J. Viewpoint invariant human re-identification in camera networks using pose priors and subject-discriminative features. IEEE Transactions on Pattern Analysis and Machine Intelligence, 37(5), pp.1095-1108, 2015.

25. Poongothai, E., Suruliandi, A. 'Features analysis of person re-identification techniques'. Int. Conf. Computing Technologies and Intelligent Data Engineering, India, 2016. BY NC) license (https://creativecommons.org/licenses/by-nc/4.0/). 